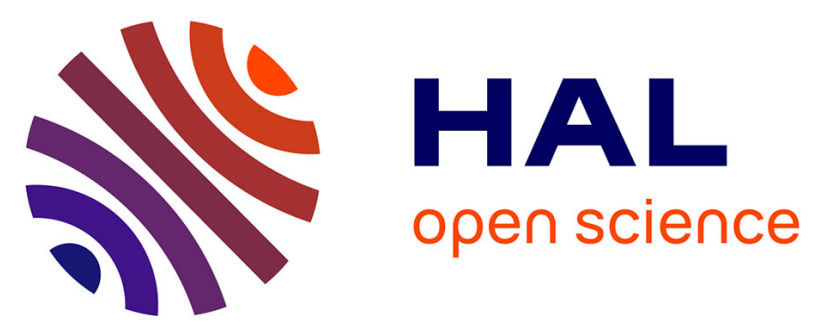

\title{
Multi-task Deep Learning based Environment and Mobility Detection for User Behavior Modeling
}

Marie-Line Alberi-Morel, Illyyne Saffar, Kamal Singh, César Viho

\section{To cite this version:}

Marie-Line Alberi-Morel, Illyyne Saffar, Kamal Singh, César Viho. Multi-task Deep Learning based Environment and Mobility Detection for User Behavior Modeling. WMLC 2019 - International Workshop on Machine Learning for Communications, Jun 2019, Avignon, France. pp.1-7. hal-02113163

\section{HAL Id: hal-02113163 https://hal.science/hal-02113163}

Submitted on 4 Jun 2019

HAL is a multi-disciplinary open access archive for the deposit and dissemination of scientific research documents, whether they are published or not. The documents may come from teaching and research institutions in France or abroad, or from public or private research centers.
L'archive ouverte pluridisciplinaire HAL, est destinée au dépôt et à la diffusion de documents scientifiques de niveau recherche, publiés ou non, émanant des établissements d'enseignement et de recherche français ou étrangers, des laboratoires publics ou privés. 


\title{
Multi-task Deep Learning based Environment and Mobility Detection for User Behavior Modeling
}

\author{
Marie Line Alberi Morel ${ }^{*}$, Illyyne Saffar* ${ }^{*}$, Kamal Deep Singh ${ }^{\dagger}$, Cesar Viho ${ }^{\ddagger}$ \\ ${ }^{*}$ Nokia Bell Labs, Nozay, France \\ ${ }^{\dagger}$ Univ Lyon, UJM Saint-Etienne, CNRS, Laboratoire Hubert Curien UMR 5516, Saint-Etienne, France \\ ${ }^{\ddagger}$ Univ Rennes, Inria, CNRS, IRISA, France \\ Email: marie_line.alberi-morel@nokia-bell-labs.com, illyyne.saffar@nokia.com, \\ kamal.singh@univ-st-etienne.fr, cesar.viho@irisa.fr
}

\begin{abstract}
Cognition of user behavior can be seen as an efficient tool for automation of future mobile networks. As a matter of fact, it amplifies the intelligence of autonomic networks in a sense that the network is more aware of the operational context. However, predicting the context of mobile users is a prerequisite for inferring the user behavior. This paper deals with the user behaviour modeling. The model includes the prediction of two main features related to mobile user context: the environment and the mobility. Practically, the question is how and where the mobile user consumes the mobile services. We investigate Deep Learning based methods for simultaneously detecting the environment and the mobility state. We empirically evaluate the effectiveness of the proposed methods using real-time radio data, which has been massively gathered from multiple diversified situations of mobile users.
\end{abstract}

Index Terms-User Behavior, Preferences, Context of use, Deep Learning, Multi-task learning, Environment, Mobility

\section{INTRODUCTION}

Future 5G mobile networks will support a large range of new services or applications, like Enhanced Mobile Broadband (eMBB), Massive Machine Type Communication (mMTC) and Ultra Reliable Low Latency Communications (URLLC). To meet the customer demands of cost-efficient services and high satisfaction, cognition of mobile user behavior is considered as a good candidate for building intelligent and suitable $5 \mathrm{G}$ autonomic networking systems. As a matter of fact, this knowledge feeds the network intelligence in the sense that the system becomes more aware of the operating context. The idea is to use cognition of mobile user behavior to enhance the self-adaptation capabilities of $5 \mathrm{G}$ networks. This will help 5G networks to face the variable consuming habits of users that impact the network conditions. This shall be achieved while individually meeting the demands or needs of customers, thus focusing on user satisfaction.

However, the awareness of the use context of mobile users is a prerequisite for inferring the mobile user behavior. It depicts all mobile user situations during which they consume mobile services or use varied applications. In [1] the use context is defined as "any information that can be used to characterize the situation of a person that is considered relevant to the interaction between a user and an application, including the user and applications themselves".
The potential value of this instance lies in its possibilities to predict noticeable trends in user behaviors/habits related to the different contexts/situations of the mobile user. As a consequence, the user behavior that defines the way a user consumes the given requested service can be seen as an abstraction of the use context.

Today, assessing the use context remains a difficult task. It is particularly problematic when the objective is to detect the personal usages or preferences automatically without requiring constant user interaction through personalized and refined questions [2]. Consequently, the reliable detection of mobile user context attributes considering multiple user-situations in a non-intrusive way, i.e., without human intervention, is an open issue that still needs to be fully addressed. Such information can help many operators to configure or optimize their networks efficiently while minimizing management and operation cost. Thus, the key idea is to automatically infer the mobile user context using Machine Learning techniques. This can be done using Deep Learning techniques that can learn in an automatic way, in real-time, and benefit from the value added by the massive data. Currently, such approaches are popular and are regarded as promising solutions. This is due to their ability to deal with complex problems and to characterize the inherent relationships between the inputs and outputs of a system without human involvement [3].

This paper, presents a preliminary study of user behavior modeling when considering the user behavior as an abstraction of the diverse usage situation experienced by mobile users. We propose to model the user behavior with the Quality-of-Experience-influencing features related to mobile user context: the environment and the mobility. Quality-ofExperience (QoE) is a metric that measures the mobile user's satisfaction depending on his or her experience of the service. The user's environment and mobility are important factors since they have a big influence on QoE. For example, a user who is indoor would experience a very different service quality as compared to those who are outdoor, all else being equal. Actually, the environment and the mobility form the conditions in which a mobile user consumes the requested services/applications. In practice, in order to estimate the environment or mobility, we have to answer the following questions: how and where a mobile user consumes the mobile 
services? Besides, these questions have to be answered at the same time (simultaneously) to detect and then predict the user behavior. Thus, to achieve it, we investigate the association of both Indoor/Outdoor Detection (IOD) and Mobility State Estimation (MSE). IOD refers to the detection of the mobile users' environments, that is to infer whether the user is Indoor or Outdoor [4]. MSE refers to the estimation whether a given user moves with low, medium or high speed. This is also specified as a requirement by LTE/5G specifications [5].

We investigate a multi-task based deep learning solution to simultaneously detect a user's environment and mobility state. This leads us to following contributions. We provide an analyses of mobile user behaviour and use context. We perform mobility state detection, which is further developed to achieve simultaneous multi-output (IOD/MSE) classification. The empirical evaluation based on real-time and highly representative $4 \mathrm{G}$ radio data, consisting of $3 \mathrm{GPP}$ signals measured by phone devices, shows the effectiveness of our approach. This data includes ground truth information and the whole dataset has been massively gathered from many diverse mobility situations and many environment types. The results also prove that the simultaneous detection of the environment as well as the mobility state estimation can be achieved with high accuracy.

The rest of this paper is organized as follows. Section II introduces the prior related work; Section III defines the user behavior and presents a discussion in terms of service usage; Section IV studies the user behavior modeling focusing on the simultaneous detection of usage environment and mobility state.

\section{RELATED WORK}

Only a few works in literature have addressed IOD and MSE using Machine Learning techniques.

The work in [6] addressed IOD using a set of signals coming from mobile phone sensors (radio signals, cell signal strength, light intensity, battery temperature ...). They proposed an IOD solution based on ML algorithms and more precisely a semi-supervised ML approach. Their solution, implemented on different android devices, shows a $92.33 \%$ of accuracy. It provides the highest detection performance in comparison to existing methods such as a supervised classifier. In [7], authors considered IOD from the network side as a classification issue. Once the indoor or outdoor location is detected, this new information further helps with other signals to localize the mobile user. For the IOD classification task, they used the radio signals $R S R P$ and $R S R Q$ signals and tested many algorithms: Support Vector Machine (SVM), logistic regression and random forest. SVM was the solution retained since it performed best. These papers show the interest of using ML approaches for IOD. Thus, this motivates us to investigate similar solutions.

Concerning mobility detection, some works already exist in literature. The work in [8] uses radio signal measurements collected under different mobility situations (low, medium, high speed). The signal attenuation is automatically estimated. This, in turn, helps to efficiently classify the mobile user

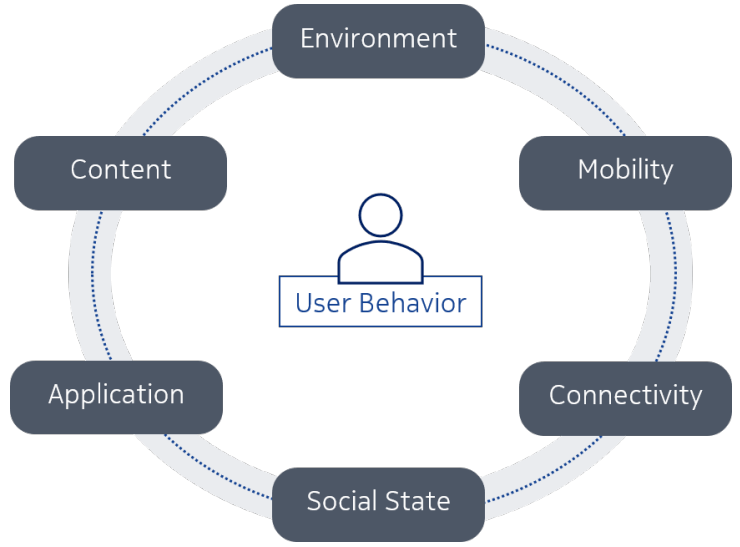

Fig. 1. User Behavior Model

environment (pedestrian, incar, non-moving) and it finally improves the handover process. In [9], authors use RSRP measurements which suffer from fading over time as a function of UE speed. They propose two methods: either based on a spectral analysis or based on a time-based spectrum spreading. In [5], the authors propose a method for estimating the UE mobility, that relies on UE history information about the UE cell sojourn time. Using such information, the eNB classifies the speed to one of the three mobility classes defined in the 3GPP standards. Both methods for mobility estimation, [9] and [5], have shown good results. Both [9] and [8] confirm that the user mobility is strongly correlated to a user's environment. In [10], authors use the knowledge extracted from simple features such as the cell ID and the sojourn time, in previously visited cells, to predict the user mobility and his future location. An interesting work has been presented in [11], where authors evaluate the user mobility in 5G networks according his behavior, his preferences and his tendencies.

To the best of our knowledge, no study has been conducted on the association of IOD and MSE using Deep Learning techniques. To efficiently estimate the user environment and mobility state at the same time, our proposed deep learning solution will use a dataset composed of radio signals as well as the data linked to power, quality and mobility.

\section{USER BEHAVIOR: MODEL AND ANALYSIS}

The context of use is defined in [ISO 13407:1999] standard as the characteristics of the users, tasks and the environment in which the system is used. This standard highlights the requirements to know not only the context of use, but also information on user (demographics), system and applications because the context affects the service/application usage situation as well as the user and the used technology. Therefore, the user behavior shall be the direct reflection of all the usage situations of mobile services.

We propose to model the user behavior by abstracting diverse usage situations experienced in the delivery zones of mobile services. For this, the model is based on the QoEinfluencing factors of user contextual information that links the 


\begin{tabular}{|l|l|l|}
\hline \multirow{4}{*}{ Context } & Environment & Indoor (home, office, café) / Outdoor (incar, suburban, urban) \\
\cline { 2 - 3 } & Mobility, speed & Walking, driving, standing, sitting, \\
\cline { 2 - 3 } & Access medium, radio interface & Wired, wireless, 3G/4G/5G, WLAN \\
\cline { 2 - 3 } & Social & Alone, with a person, with a group \\
\hline \multirow{2}{*}{ Application } & Type & VoIP, live or non live service, Web access, VoD with short/full movies, on-line gaming \\
\cline { 2 - 3 } & Content & Action movie, interview, video-conference or video call, image, synthesis \\
\hline
\end{tabular}

TABLE I

QUALITY-OF-EXPERIENCE-INFLUENCING FACTORS OF CONTEXTUAL INFORMATION

usage situation and the application. The contextual information is summarised in Table I.

The contextual information is classified into two main categories of QoE influencing factors: use context and application. The use context includes the environment, with more refined information like indoor/outdoor location (instead of only the exact user coordinates), device speed and orientation, the social context, i.e., people around the user and the type of access medium. The applications used on mobile devices are diverse. They include communication, web, social network applications, multimedia streaming, TV channels applications and also mobile apps [12]. The user behavior depicted in Figure 1 is then modeled as a multi-attribute entity. Each attribute is directly linked to the service usage and is defined as follows:

- Environment (indoor, outdoor ...),

- Mobility (low, high ...),

- Connectivity $(3 \mathrm{G}, 4 \mathrm{G}, 5 \mathrm{G}, \ldots)$,

- Social state (alone or in a group)

- Application (conversational, video, ...)

- Content (news, sport, action movies, ...)

Contrary to traditional TV users who just watch scheduled programs, mobile service users are free to choose the content they want, at any point in time and space. During a day, a user can be in different situations, such as walking outdoor, in a car, at the work office, in a mall, in a café or at home. As a matter of fact, mobile users' preferences for certain applications or contents is linked with the usage situations [13]. Statistical studies show that mobile phones are mostly used in a building for internet service $(80 \%)$ and for a call $(70 \%)$ [14]. This can be explained by the fact that the different use contexts pose their own limitations, which in turn impact the potential application usages. In [12], [15], [2], the most commonly mentioned physical environments of application usage are again indoors (waiting halls or lounges, work, home and cafes), but also include vehicles, such as public transportation and private cars. In motion, audio is the preferred media, whereas, during stationary reception, text and video are the most pleasant media. For information assimilation, the café environment is preferred, while the bus or car environment is not that preferred. This is explained by the calm and the pleasant atmosphere of a café which is suitable for focusing on viewing. In the bus context, people may focus their attention to, e.g., watching mobile $\mathrm{TV}$, but the complicated task on the move results generally in an unpleasant experience of entertainment.
Consequently, the mobile devices are mostly used in indoor or in-car context. The environment and mobility are therefore important factors for describing the mobile user behavior when he is experiencing a service. In the following, we investigate the prediction of the user behavior when connecting to cellular networks and experiencing an application. The prediction will focus only on two QoE-influencing features, the environment and the mobility state.

\section{USE CASE ANALYSIS: ENVIRONMENT AND MOBILITY DETECTION}

In this section, we study the simultaneous detection of user environment (Indoor, Outdoor) and mobility state (Low, Medium, High) using a deep learning approach.

\section{A. Why Multi-Task Deep Learning?}

In machine learning, we use the concept of Multi-Task Learning (MTL), when a single model is used to solve a series of related tasks (by task we mean: regression, prediction, etc.). The idea of MTL is inspired from human learning activities. As humans, we often use the same basics that provide us with the necessary skills to learn several tasks and master more complex techniques. For example, we use the algorithmic and the theoretic knowledge, which serves as a base acquired at school, to further learn different programming language.

Generally, to guarantee good performances while training a Multi-Task model, we should ensure that there is a correlation between the different tasks. Indeed, correlation between tasks means that some relation exists among the tasks and capturing such information can result in better performance, as compared to learning each task independently [16] [17].

Works in machine learning treat IOD and MSE tasks independently. However, in practice, analyzing the user behavior requires simultaneously processing both the tasks since they both impact the user behavior. The idea of this paper is to predict the user behavior by detecting the environment and the mobility state at the same time. This requires to simultaneously perform MSE and IOD using an approach based on multitask deep learning (DL-MTL). Actually, DL-MTL for the user behavior context can provide a better generic model rather than studying every specific behavior separately. When for a given single task the features are not sufficient, or very noisy to the point that the model can not quantify its relevance, then DL-MTL can help solving this issue. DL-MTL can do that by using additional features intended for other tasks to clarify and provide additional information and evidence for its relevance or irrelevance [18], [19]. 


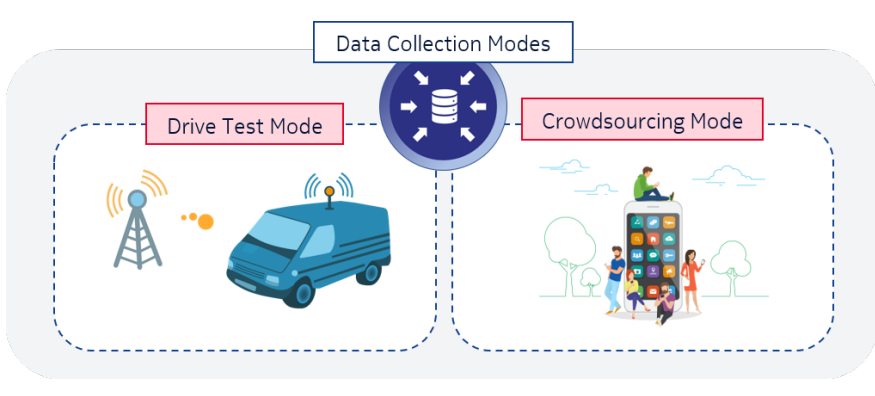

Fig. 2. Data collection modes

\section{B. Dataset Description}

To use Deep Learning, it is useful to have massive input data. Furthermore, deep learning is used for the problems where modeling relationships between large number of parameters is not tractable. This is the case in our problem because we simultaneously infer both the attributes of environment and mobility. The model has to extract, at same time, the complexity of the variety of situations imposed by all the available combinations generated by the two attributes. Actually there are two ways to collect data (Figure 2) :

- Drive test mode: this mode is widely used to collect data. It is a mode in which a vehicle or people equipped with sensors move in specific places to collect data. However, this controlled mode imposes limits on capturing the reality. Such data collection campaigns are run for limited hours per day during short periods (couple of weeks) and at some specific places. For user behavior prediction with DL-MTL approach, using data collected in drive test mode will not deliver a generic model, since, we will miss big sides of users' behavior, for example the case of his night life, etc.

- Crowdsourcing mode: Recently surfaced with big firms like Google and Netflix, the crowdsourcing mode allows to gather data more cheaply and in large quantities, more than ever before. But most importantly, it allows to better reflect the users' behaviors by capturing a huge diversity of their experiences. In this mode, the collected data consists of signals measured by the mobile phones. This data is gathered and controlled by the network of a tenant (e.g. service provider or application provider) in order to mine personal information. To be able to do this, first users should agree and should themselves install the necessary software.

In our case, we propose to collect data for the training phase using a crowdsourcing mode controlled by the operator. This mode allows us to gather data inside the network and corresponding to UE-specific radio measurements and additional data required to do the labelling and then to train the model in supervised mode (Figure 3). This, in turn, improves the machine learning performance because with this data collection mode, we get more highly representative data.

For our study, we are using a dataset that has been collected in a crowdsourced mode. With this campaign of data measurement, we try to build a dataset as close as possible to the complexity and the variety of several locations in France visited by mobile user moving in real world. Figure 4 shows the places where our dataset has been collected. Every red point corresponds to a visited place (and implicitly the roads linking two visited points). The collection duration corresponds to 16 months with an average $24 h / 7$ with an average of 1 measurement per 15 seconds while the mobile phone session is active and 1 measurement per 2 minutes otherwise. For 16 months of massive collection the measurements are related to many different and diversified environments like mountain, beach, forest, companies, cafes, streets, bars, parks, restaurants, lakes, etc. This includes many cities and places like countryside, villages, small cities, metropolis, and different countries, but for this paper we are only studying the data collected in France. Thanks to this long data collection period, the data has been collected in every type of weather such as rainy, sunny, snow, etc.

\section{Environment and Mobility Detection system: architecture and implementation}

We aim to compare the system with two independent classification tasks versus the one with two simultaneous tasks. The system performing simultaneous tasks uses similar input signals, whereas, the system with independent tasks uses separate input signals for each task. There exist many methods in literature for MTL with deep learning: parameters sharing, feature sharing,... [19], [18]. We are interested in the most used approach which is a classical method of MTL consisting in sharing hard parameters. MTL with sharing of hard parameters consists on sharing the first hidden layers between the tasks. Then some specific layers are specified for every task. Using this method reduces often the overfitting risk. Thus, we propose the MTL system as described in Figure 5. It is composed of three main parts :

- Input: A first input layer fed with shared features for both IOD and MSE tasks treated simultaneously. These inputs consists in of 7 main signals. Recall that we focus

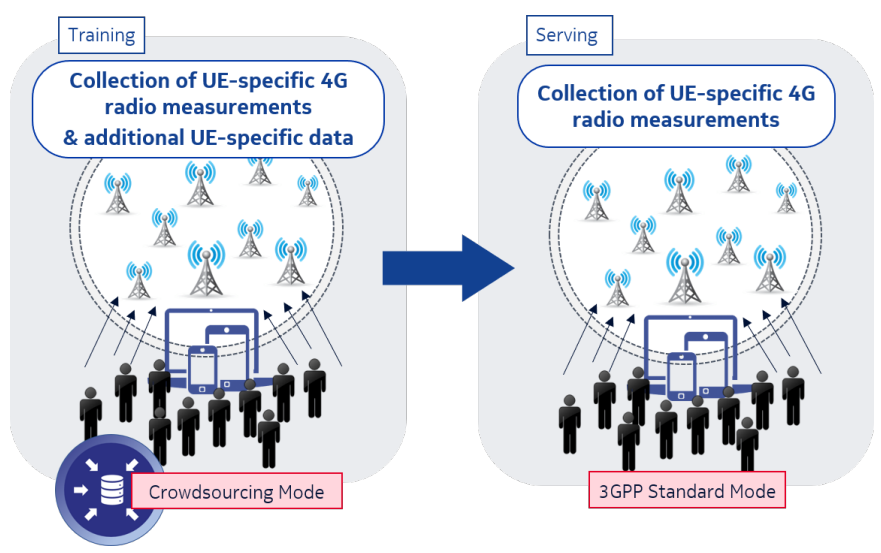

Fig. 3. Data collection scheme for training and serving phases 


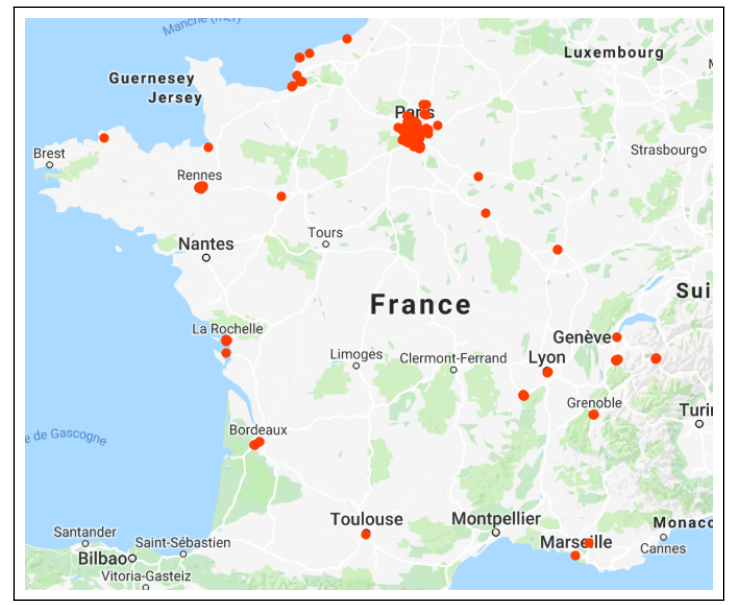

Fig. 4. Data collection points in France: multiple environments and places

on features that can be collected in the $4 \mathrm{G}$ infrastructure side.

- RSRP: Average received power of the Reference Signal (RS). The RSRP value lies between $-140 \mathrm{dBm}$ to $-44 \mathrm{dBm}$

- CQI: Channel Quality Indicator that is used to indicate the most appropriate transmission modulation and coding scheme to be used

- TA: Timing Advance is used to control UL signal transmission timing

- MI: The number of the Cell ID changes (NCID) in a sliding window of a given duration (TCRmax)

- ST: Sojourn time in a cell

- Extra Signals : signals derived from $R S R P$ and $T A$

- Label: Indoor or Outdoor label for the IOD \& Low, Medium, High mobility label for the MSE

- Core : Containing 2 main blocks: (1) first block for feature extraction. (2) second block of hidden layers.

- Output : An output layer for IOD (2 Classes: Indoor, Outdoor) and MSE (3 Classes: Low, Medium, High).

DL-MTL is implemented with both Scikitlearn and Keras in python. We tune Neural Network hyper-parameters using a GridSearch algorithm (which simply is an exhaustive search through a manually specified subset of the hyper-parameters for Deep Learning). The DL-MTL module is then a feed forward neural network composed of an input layer, an output layer, 5 fully connected hidden layers. We added also 2 dropout layers to regularize and minimize the over-fitting.

The compared system which implements independent tasks is explained as follows. IOD task is done using the input features and a supervised Deep Learning algorithm described in [4]. MSE task uses a part of features described above and use a similar Deep Learning algorithm.

\section{Results and discussion}

The training is done using the labeled part of our dataset. For training, we used $(70 \%)$ of the labeled data and we used the $(30 \%)$ remaining for the model performance evaluation.
DL-MTL shows good performance in terms of accuracy when trained on our crowdsourced dataset: $97.21 \%$ for IOD and $93.18 \%$ for MSE. However, the accuracy alone can not quantify the overall performance even if faced with the issue of unbalanced data. Indeed, preliminary statistics of our data show that the data proportion between indoor and outdoor classes is unbalanced. For the IOD task, as shown in the figure 6, we have $75 \%$ Indoor instances vs. $25 \%$ Outdoor instances. For the MSE task, we have $90 \%$ Low vs. $5 \%$ Medium vs. High $5 \%$ instances.

This issue of unbalanced data is not related to the data collection constraints, but it is rather due to the real human activity. Effectively, users are more static and indoors rather than in mobility situation or outdoors. Moreover, users prefer to use their mobiles in specific situations. Thus, we also use F1-score metric in addition to the accuracy for performance evaluation. F1-score is one of the most used metric in case of unbalanced data classes. F1-score metric by definition is the weighted average of Precision and Recall according to the following relation:

$$
F 1-\text { score }=2 . \frac{\text { Precision } . \text { Recall }}{\text { Precision }+ \text { Recall }}
$$

where Precision is the number of correct positive results divided by the number of all positive results returned by the classifier, and Recall is the number of correct positive results divided by the number of all relevant samples.

\begin{tabular}{|l|c|c|}
\hline & F1-score with DL & F1-score with DL-MTL \\
\hline IOD & $92.81 \%[4]$ & $\mathbf{9 7 . 1 9} \%$ \\
\hline MSE & $78.30 \%$ & $\mathbf{8 7 . 6 9} \%$ \\
\hline
\end{tabular}

TABLE II

ENVIRONMENT AND MOBILITY SUPERVISED CLASSIFICATION PERFORMANCE: F1-SCORE VS. DL ARCHITECTURE \& DL-MTL ARCHITECTURE

Resolving the tasks simultaneously can optimize the user behavior model in general. As shown in Table II, IOD and MSE have better performance with a DL-MTL architecture. As seen in Table II, the performance gain for MSE task is approx. $10 \%$. Note that this performance improvement was obtained without changing any other parameter like input features, data etc. The correlation between the environment and the mobility has been beneficial for both the tasks. With such structure they have shared data inputs that brought to each others meaningful additional information (for example a user can't be indoor with medium or high speed). Moreover, the extra output contributes further in fine tuning the neural network parameters during training. That is why DL-MTL is showing better performance.

For our work, we consider that $5 \%$ of error is tolerable that is to say an F1-score of $95 \%$. This is inspired from network dimensioning requirement. Indeed, while mobile networks dimensioning, an error up to $5 \%$ is qualified as an admissible error rate. The first task delivers performance above the fixed threshold, however the MSE is still under $95 \%$ of F1-score. 


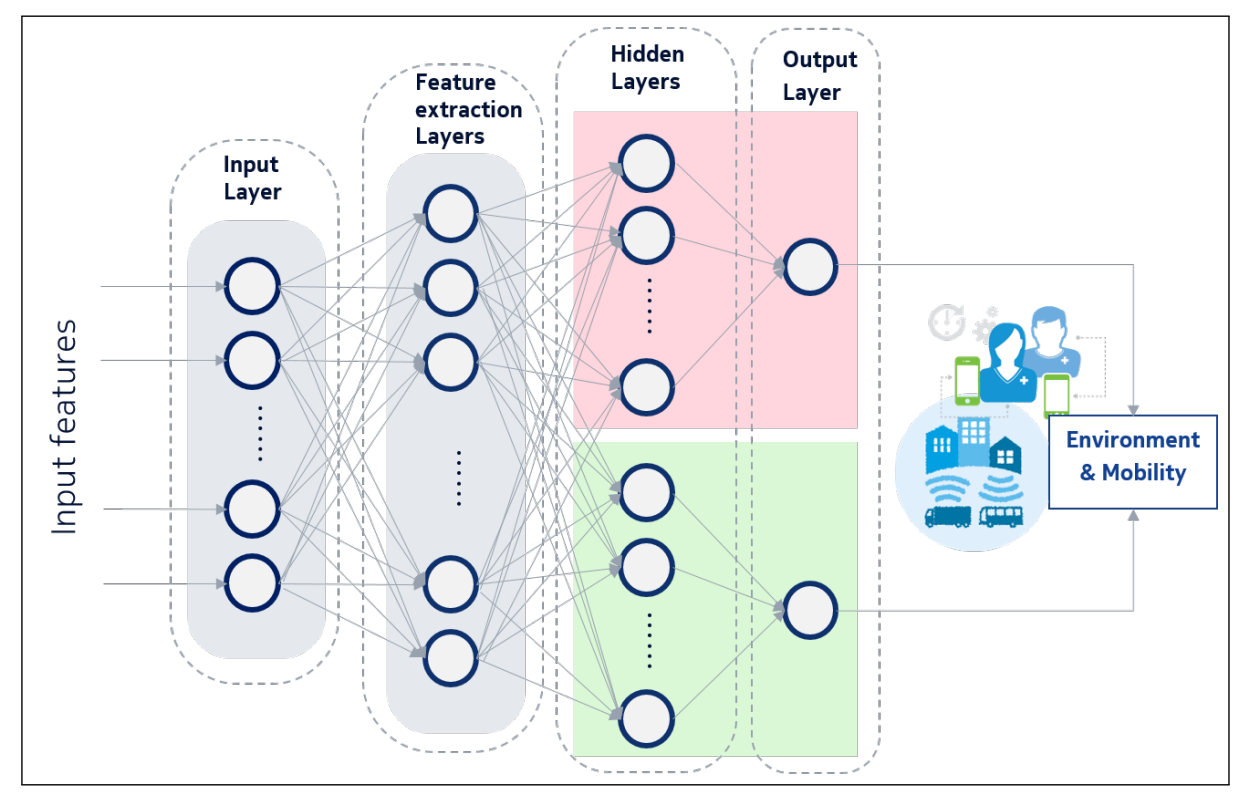

Fig. 5. Multi-Task Learning using Deep Learning architecture. Note that there are several hidden layers and not just one as it may initially appear.

Considering this, the IOD task performance achieves a good performance, but MSE still needs to be improved.

The confusion matrix for MSE illustrated in Figure 7 shows that the model is classifying well the Low mobility class (with more than $90 \%$ of "true positives"), but relatively less well for the Medium and High mobility classes (with less than $75 \%$ of "true positives"). This is explained by the fact that the dataset is unbalanced as shown in Figure 6 that depicts the mobility state distribution per environment. This challenge is inherent to the user behavior: people are mostly in static condition than in high speed. Moreover, as soon as the user speed increases, the network quality deteriorates and consequently the user switches to $3 \mathrm{G}$ or even to GSM (sometimes). These two phenomena result in less data collected in these scenarios. For future work, we intend to investigate further to solve the issue due to the lack of balance between the mobility classes by adding other relevant features for training. The objective will be to help DL-MTL to detect the Medium and the High

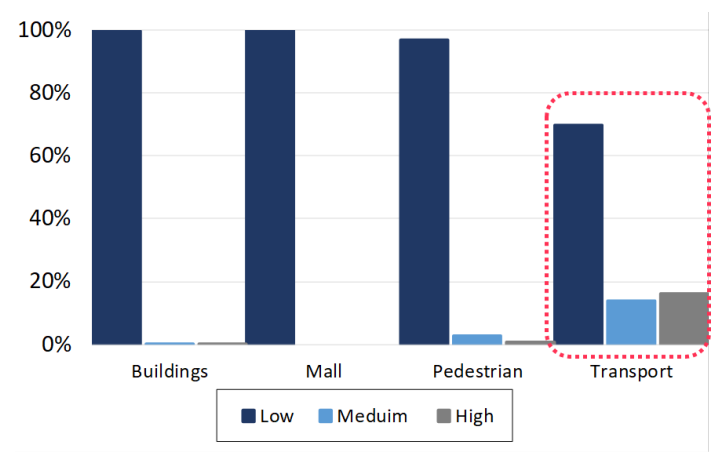

Fig. 6. Mobility State distribution per Environment classes more efficiently.

\section{CONClusion}

The use context has a great importance for tracking user's behavior or preferences. The personalization shall be done for multiple contexts of use and thus, address multiple users and usage situations.

Thus, in this paper, we focused on modeling user behaviour and the use context. Two important elements of user behaviour and use context were considered: environment (indoor or outdoor) and mobility (low, medium or high). These tasks named as indoor/Outdoor detection (IOD) and Mobility State Estimation (MSE) were performed using deep learning. We used a real massive data-set consisting of input signals obtained from the mobile network.

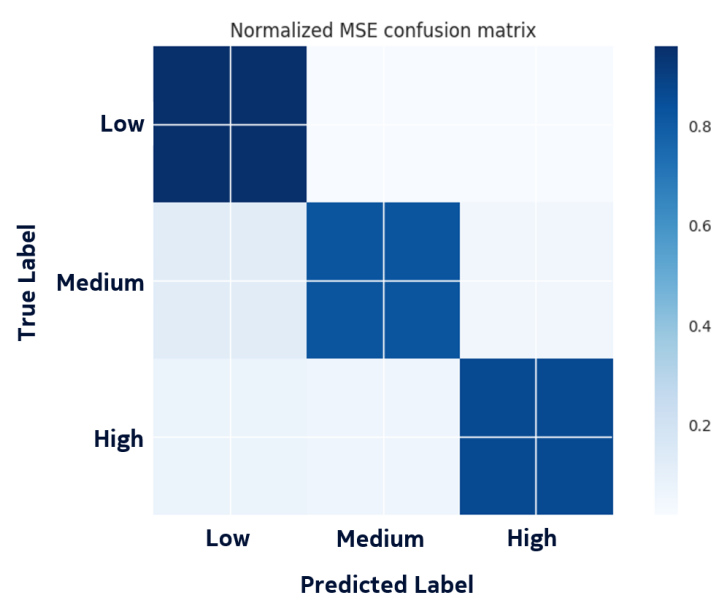

Fig. 7. Confusion Matrix for the MSE Task - Multi-Task Learning case 
In terms of contributions, we provided a discussion on mobile user behavior and use context. Then, a deep learning and data based approach was used for mobility state estimation, which was finally developed into a module that simultaneously performed the IOD and MSE tasks. Notably, this module (called DL-MTL) showed better performance as compared to another approach which performed these tasks independently. In future, we plan to focus on improving the results of MSE task. We will also focus on tackling the issues of unbalanced data and validating our model considering many more varieties of user profiles.

\section{REFERENCES}

[1] S. Husnjak, D. Perakovic, I. Forenbacher and I. Jovovic, Identification and Prediction of User Behavior Depending on the Context of the Use of Smart Mobile Devices, 26th DAAM International Symposium on Intelligent Manufacturing and Automation, 2016

[2] E. Kaasinen, User Acceptance of Mobile Services - Value, Ease of Use, Trust and Ease of Adoption, 2005

[3] M. Wang, Y. Cui, X. Wang, S. Xiao, and J. Jiang, Machine Learning for Networking: Workflow, Advances and Opportunities, IEEE Network Accepted for publication, 2017

[4] Illyyne Saffar, Marie-Line Alberi Morel, Kamal Deep Singh and César Viho, Machine Learning with partially labeled Data for Indoor Outdoor Detection. In : IEEE Annual Consumer Communications \& Networking Conference (CCNC). 2019.

[5] HERCULEA, D., CHEN, C. S., HADDAD, M., et al. Straight: Stochastic geometry and user history based mobility estimation. In : Proceedings of the 8th ACM International Workshop on Hot Topics in Planet-scale mObile computing and online Social neTworking. ACM, 2016. p. 1-6.

[6] RADU, Valentin, KATSIKOULI, Panagiota, SARKAR, Rik, et al. A semi-supervised learning approach for robust indoor-outdoor detection with smartphones. In : Proceedings of the 12th ACM Conference on Embedded Network Sensor Systems. ACM, 2014. p. 280-294.
[7] RAY, Avik, DEB, Supratim, et MONOGIOUDIS, Pantelis. Localization of LTE measurement records with missing information. In : Computer Communications, IEEE INFOCOM 2016-The 35th Annual IEEE International Conference on. IEEE, 2016. p. 1-9.

[8] ALAYA-FEKI, Afef Ben Hadj, LE CORNEC, Alain, et MOULINES, Eric. Optimization of Radio Measurements Exploitation in Wireless Mobile Networks. JCM, 2007, vol. 2, no 7, p. 59-67.

[9] HADDAD, Majed, HERCULEA, Dalia Georgiana, ALTMAN, Eitan, et al. Mobility state estimation in LTE. In : Wireless Communications and Networking Conference (WCNC), 2016 IEEE. IEEE, 2016. p. 1-6.

[10] MICHAELIS, Stefan. Balancing high-load scenarios with next cell predictions and mobility pattern recognition. Universitätsbibliothek Dortmund, 2012.

[11] GE, Xiaohu, YE, Junliang, YANG, Yang, et al. User mobility evaluation for $5 \mathrm{G}$ small cell networks based on individual mobility model. IEEE Journal on Selected Areas in Communications, 2016, vol. 34, no 3, p. 528-541.

[12] V. A. Siris, K. Balampekos and M. K. Marina, Mobile Quality of Experience: Recent Advances and Challenges, Workshop on Information Quality and Quality of Service for Pervasive Computing, 2014

[13] S. Ickin et al., Factors influencing quality of experience of commonly used mobile applications, IEEE Communications Magazine, vol. 50, no. 4, pp. 48-56, 2012.

[14] http://www.hetnetforum.com/, HetNet Forum, 2018

[15] T. Soikkeli, J. Karikoski, H. Hämmäinen, Diversity and End User Context in Smartphone Usage Sessions, Next Generation Mobile Applications, Services and Technologies (NGMAST), 5th International Conference, 2011, pp. 7-12.

[16] LI, Changsheng, YAN, Junchi, WEI, Fan, et al. Self-Paced Multi-Task Learning. In : AAAI. 2017. p. 2175-2181.

[17] XU, Donna, SHI, Yaxin, TSANG, Ivor W., et al. A Survey on Multioutput Learning. arXiv preprint arXiv:1901.00248, 2019.

[18] ZHANG, Yu et YANG, Qiang. A survey on multi-task learning. arXiv preprint arXiv:1707.08114, 2017

[19] RUDER, Sebastian. An overview of multi-task learning in deep neural networks. arXiv preprint arXiv:1706.05098, 2017. 